\title{
The association of elevated circulating endocan levels with lung function decline in COPD patients
}

This article was published in the following Dove Press journal:

International Journal of COPD

\author{
Luqi Dai ${ }^{1,2, *}$ \\ Junyun $\mathrm{He}^{3, *}$ \\ Jun Chen ${ }^{1,2}$ \\ Tao Wang ${ }^{1,2}$ \\ Lian Liu ${ }^{1,2}$ \\ Yongchun Shen ${ }^{2}$ \\ Lei Chen ${ }^{2}$ \\ Fuqiang Wen ${ }^{1,2}$ \\ 'Division of Pulmonary Diseases, State \\ Key Laboratory of Biotherapy, West \\ China Hospital, West China School of \\ Medicine, Sichuan University, Chengdu, \\ Sichuan 6I004I, China; '2Department \\ of Respiratory and Critical Care \\ Medicine, West China Hospital, West \\ China School of Medicine, Sichuan \\ University, Chengdu, Sichuan 6I004I, \\ China; ${ }^{3}$ Department of Respiratory \\ Medicine, Hospital of Chengdu \\ Office of People's Government of \\ Tibetan Autonomous Region of China, \\ Chengdu, Sichuan 6I004I, China \\ *These authors contributed equally \\ to this work
}

\begin{abstract}
Background: Endocan is thought to be a novel inflammatory marker that is associated with a variety of inflammatory diseases. However, its role in the pathogenesis of COPD remains unclear. This study aims to explore the potential role of endocan in COPD.

Methods: In total, 27 healthy volunteers, 55 COPD patients and 36 acute exacerbation of chronic obstructive pulmonary disease (AECOPD) patients were included in the study. Basic demographic characteristics, clinical features and blood samples were collected. Magnetic luminex screening assays were used to detect the concentration of endocan, Fas and Fas ligand (Fas-L) in plasma. Differences between groups were compared using an Independent sample $t$-test, Welch's $t$-test, chi-squared test and Wilcoxon rank sum test. The correlations of plasma endocan with lung function parameters, Fas and Fas-L were analyzed by Pearson's partial correlation test (adjusted for age, gender, body mass index and smoking history) and multiple linear regression.

Results: Plasma endocan levels in COPD patients were significantly higher than those in healthy volunteers $(509.7 \pm 18.25 \mathrm{pg} / \mathrm{mL}$ vs $434.8 \pm 18.98 \mathrm{pg} / \mathrm{mL}(P=0.0124))$, and AECOPD patients had the highest levels of endocan $(524.7 \pm 27.18 \mathrm{pg} / \mathrm{mL})$. Correlation analysis showed that circulating endocan had a negative correlation to $\mathrm{FEV}_{1} / \mathrm{FVC}, \mathrm{FEV}_{1} /$ predictive and $\mathrm{FVC}$ (adjusted $r=-0.213, P=0.03$; adjusted $r=-0.209, P=0.034$; and adjusted $r=-0.300, P=0.002$, respectively), and had a positive correlation to Fas (adjusted $r=0.280, P=0.004$ ).

Conclusion: Our study shows that elevated circulating endocan levels are associated with reduced lung ventilation function in COPD and AECOPD patients. In addition, endocan may influence apoptosis in COPD, suggesting that endocan may play a role in COPD pathogenesis.
\end{abstract}

Keywords: endocan, COPD, lung function, apoptosis

\section{Introduction}

COPD, a chronic disease that is characterized by airflow limitation and affects the airway and lung parenchyma, is a global disease closely related to smoking and is expected to become the fifth leading cause of disability. ${ }^{1,2}$ Acute exacerbation of COPD is very common and seriously affects quality of life and is the main cause of death in COPD patients. ${ }^{3,4}$ Pulmonary vascular endothelial cell damage and vascular inflammation are involved in the pathogenesis of COPD. Pulmonary inflammation can also spread to the systemic circulation and can increase the occurrence of cardiovascular events. ${ }^{5,6}$ Endothelial cells play an important role in acute inflammation related to COPD exacerbation. ${ }^{7-9}$ However, it is not yet clear what role endothelial cells play in COPD and what factors are involved.

Endocan, formerly known as endothelial cell-specific molecule 1 (ESM-1), is an endothelial-associated proteoglycan that is secreted mainly by activated endothelial cells. It is preferentially expressed in vascular endothelial cells in the lung and kidney,
Correspondence: Fuqiang Wen; Lei Chen Department of Respiratory and Critical Care Medicine, West China Hospital, West China School of Medicine, Sichuan University, Guoxuexiang 37, Chengdu, Sichuan 61004I, China Email wenfuqiang.scu@gmail.com; Ichens@I26.com 
and its overexpression can be seen in some neonatal and proliferating tissues as well as some tumors. ${ }^{10,11}$

Recent studies have shown that endocan is associated with endothelial dysfunction and regulates inflammation in patients with inflammatory diseases, such as sepsis and chronic kidney disease. ${ }^{12,13}$ Thus, endocan is considered as a novel inflammation-related factor. ${ }^{14,15}$ The interaction of endocan with lymphocyte function-associated antigen-1 inhibits leukocytes from binding to the vascular endothelium. ${ }^{10,16}$ Multiple cytokines, including TNF-A, interleukin-1, microbial lipopolysaccharide and angiogenic factors can upregulate endocan expression, while interferon- $\gamma$ seems to inhibit it. ${ }^{17,18}$ Endocan can relieve the apoptosis of lung epithelial cell in acute lung injury (ALI) by releasing over-activated mitochondrial unfolded protein response; endocan also promotes the differentiation and apoptosis of gastric adenocarcinoma cell by activating caspase- and p53-dependent apoptotic pathway. ${ }^{19,20}$

Therefore, we proposed that endocan may participate in the inflammatory activity and abnormal apoptosis of COPD. In this study, we aimed to evaluate the plasma levels of endocan in healthy subjects and patients with COPD, and to compare the correlation between endocan and changes in lung function and apoptosis.

\section{Methods}

\section{Inclusion and exclusion criteria}

The research protocol complied with the principles of the Declaration of Helsinki and was approved by the Ethics Committee of the West China Hospital of Sichuan University of the People's Republic of China. All subjects provided written informed consent. From January to December 2016, the healthy volunteers, COPD patients and those with exacerbation of COPD were recruited at the hospital outpatient and inpatient departments of West China Hospital of Sichuan University. All patients were previously diagnosed with COPD based on GOLD documents. All subjects were asked about their medical history in detail, and chest CTs from the most recent year were reviewed. Exclusion criteria were as follows: 1) suffering from diseases other than COPD or acute exacerbation of chronic obstructive pulmonary disease (AECOPD), 2) unable or unwilling to cooperate with a doctor, 3 ) unable or unwilling to perform lung function tests, and 4) suffering from pulmonary diseases (acute respiratory distress syndrome [ARDS], asthma and lung cancer) or other diseases known to affect plasma endocan (including tumors, atherosclerosis and chronic kidney disease).

\section{Collection of demographic and clinical characteristics}

Basic information such as name, gender, ethnicity, age and smoking history was collected for all subjects. All the subjects underwent lung function test, administered by professionals, in the pulmonary function room, and the function data were collected. To avoid bias, the researchers who conducted spirometry testing did not know the patient's enrollment beforehand.

\section{Measurement of endocan, Fas and Fas ligand}

Blood samples were collected from subjects the morning after enrollment. All subjects fasted from 9:00 pm the night before. Venous blood was collected from the median cubital vein and plasma was separated and stored immediately at $-80^{\circ} \mathrm{C}$ until analysis. The endocan, Fas and Fas ligand (Fas-L) levels in the plasma were measured using the Magnetic Luminex Screening Assay (R\&D Systems, Minneapolis, MN, USA) on a Bio-Plex 200 (Bio-Rad, Hercules, CA, USA) in the Division of Laboratory of West China Hospital. The detection sensitivity of endocan, Fas and Fas-L were $1.08 \mathrm{pg} / \mathrm{mL}$, $3.23 \mathrm{pg} / \mathrm{mL}$ and $1.23 \mathrm{pg} / \mathrm{mL}$, respectively.

\section{Statistical analysis}

All data were analyzed using SPSS 22.0 for Windows (IBM, Chicago, IL, USA) and figures were created using GraphPad Prism 6.01 for Windows (GraphPad Software Inc, La Jolla, CA, USA). For quantitative data, Shapiro-Wilk tests were used to determine whether they obey the normal distribution. Differences between groups were analyzed by independent sample $t$-test, $t$ ' test, chi-squared test or Wilcoxon rank sum test. The correlations between plasma biomarkers and lung function parameters were analyzed using the Pearson's partial correlation test to correct for age, gender, body mass index (BMI) and smoking history. Multiple linear regression analysis was conducted to confirm the aforementioned relationships. All data are presented as mean $\pm \mathrm{SD}$, and $P$-values $<0.05$ were considered statistically significant.

\section{Results Subject characteristics}

In total, 118 subjects including 27 healthy controls, 55 COPD patients and 36 AECOPD patients with available lung function parameters and endocan levels were enrolled in the study. Among the 27 healthy controls, 55 COPD patients and 36 AECOPD patients, 8, 43 and 20 had a history of smoking, respectively. We applied the Global Initiative for Chronic 
Obstructive Lung Disease (GOLD) to grade airflow limitation in patients. In total, 19 subjects were classified as GOLD 1, 40 as GOLD 2, 23 as GOLD 3 and 9 as GOLD 4. There were no statistical differences in gender, BMI or number of current smokers and previous smokers between the three groups; however, healthy controls were younger than the COPD and AECOPD groups $(P<0.05)$. Compared with healthy controls, FVC, $\mathrm{FEV}_{1}, \mathrm{FEV}_{1} /$ Predictive FEV $_{1}\left(\mathrm{FEV}_{1} / \mathrm{Pre}\right)$ and $\mathrm{FEV}_{1} / \mathrm{FVC}$ were significantly lower in COPD and AECOPD patients $(P<0.05)$. Table 1 shows the characteristics of these three groups.

\section{Increased plasma endocan levels in patients with COPD and AECOPD}

Our analyses showed that endocan levels in COPD patients $(509.7 \pm 18.25 \mathrm{pg} / \mathrm{mL})$ and AECOPD patients $(524.7 \pm 27.18 \mathrm{pg} / \mathrm{mL})$ were higher than those in healthy controls $(434.8 \pm 18.98 \mathrm{pg} / \mathrm{mL})(P=0.0124$ and $P=0.0139$, respectively, Figure 1A). No statistical difference was found between COPD patients and AECOPD patients. The average endocan level was $447.0 \pm 23.58 \mathrm{pg} / \mathrm{mL}(\mathrm{n}=19)$ for healthy nonsmokers, $405.8 \pm 30.82 \mathrm{pg} / \mathrm{mL}(\mathrm{n}=8)$ for healthy smokers, $517.2 \pm 45.03 \mathrm{pg} / \mathrm{mL}(\mathrm{n}=12)$ for COPD nonsmokers, 507.6 $\pm 19.97 \mathrm{pg} / \mathrm{mL}(\mathrm{n}=43)$ for COPD smokers, $509.1 \pm 40.78 \mathrm{pg} / \mathrm{mL}(\mathrm{n}=16)$ for AECOPD nonsmokers and $537.2 \pm 37.16 \mathrm{pg} / \mathrm{mL}(\mathrm{n}=20)$ for AECOPD smokers. Endocan levels in COPD and AECOPD smokers were higher than those in healthy smokers $(P=0.0405$ and $P=0.0449$, respectively, Figure 1B). AECOPD smokers had significantly elevated endocan levels when compared with healthy nonsmokers $(P=0.049$, Figure 1B).

\section{Correlations between endocan and pulmonary function parameters, Fas and Fas-L}

After adjusting for age, gender, BMI and smoking history, we found that plasma endocan in all healthy people and COPD patients was negatively correlated with $\mathrm{FEV}_{1} / \mathrm{FVC}$ (adjusted $r=-0.251, P=0.036$, Figure $2 \mathrm{~A}$ ), $\mathrm{FEV}_{1} /$ Pre (adjusted $r=-0.244, P=0.042$, Figure 2B) and FVC (adjusted $r=-0.263$, $P=0.028$, Figure $2 \mathrm{C}$ ). We also found that endocan levels were positively correlated with Fas (adjusted $r=0.318, P=0.007$, Figure 2D); however, there was no obvious correlation between endocan and Fas-L (adjusted $r=0.101, P=0.404$ ). In addition, endocan in all subjects was correlated with $\mathrm{FEV}_{1} / \mathrm{FVC}$ (adjusted $r=-0.213, P=0.03$, Figure $3 \mathrm{~A}$ ), $\mathrm{FEV}_{1} /$ Pre (adjusted $r=-0.209, P=0.034$, Figure 3B), FVC (adjusted $r=-0.300, P=0.002$, Figure $3 \mathrm{C}$ ) and Fas (adjusted $r=0.280$,
$P=0.04$, Figure 3D), and the correlation between endocan with Fas-L was not found (adjusted $r=0.146, P=0.140$ ).

\section{Multiple linear regression analysis}

Multiple linear regression analysis showed that age, BMI, gender, smoking history, $\mathrm{FEV}_{1} / \mathrm{FVC}$, Fas and Fas-L were the independent parameters associated with endocan (Table 2), and age, $\mathrm{FEV}_{1} / \mathrm{FVC}$, Fas and Fas-L were statistically significant in this model $(P<0.05)$.

\section{Discussion}

This study was the first to explore the role of endocan in COPD. We found that endocan levels in COPD and AECOPD patients were significantly higher than those in healthy controls. Endocan level was negatively correlated with $\mathrm{FEV}_{1} / \mathrm{FVC}, \mathrm{FEV}_{1} /$ Pre and FVC, and was positively correlated with Fas, suggesting that endocan may be a useful biomarker for COPD.

We found that the endocan levels of COPD patients were significantly higher than those of healthy controls. While changes in pulmonary vascular structure and function are involved in the pathophysiological changes of COPD, ${ }^{21}$ the specific mechanism underlying the changes remains unclear. Endocan is a soluble dermatan sulfate proteoglycan produced by activation of the endothelium. It regulates cell proliferation, differentiation, migration and adhesion through various means and is a novel inflammatory marker. ${ }^{18}$ Endocan can influence the development of ALI and ARDS and may play an important role in predicting the prognosis of ARDS patients. ${ }^{22-24}$ Our work shows that endocan may also play a role in the inflammatory response of COPD.

In addition, our results show that endocan levels are negatively correlated with $\mathrm{FEV}_{1} / \mathrm{FVC}, \mathrm{FEV}_{1} /$ Pre and FVC. Endocan is mainly expressed in the pulmonary microcirculation where it plays an important role in the maintenance of endothelial homeostasis and is closely related to inflammation. ${ }^{17}$ Elevation of COPD airway inflammatory factors may be associated with endothelial dysfunction, and endothelial damage is proportional to the severity of bronchial obstruction. ${ }^{32,33}$ Our study demonstrates that endocan links airway obstruction with endothelial system changes. Thus, endocan can be used as a simple indicator of $\mathrm{FEV}_{1} / \mathrm{FVC}, \mathrm{FEV}_{1} /$ Pre and FVC in patients with COPD or AECOPD, especially under circumstances in which lung function tests cannot be performed.

Endocan was also positively correlated with Fas. Fas is a type I transmembrane glycoprotein belonging to the tumor necrosis factor receptor and the nerve growth factor receptor superfamily. When Fas binds to the Fas-L, it recruits several 


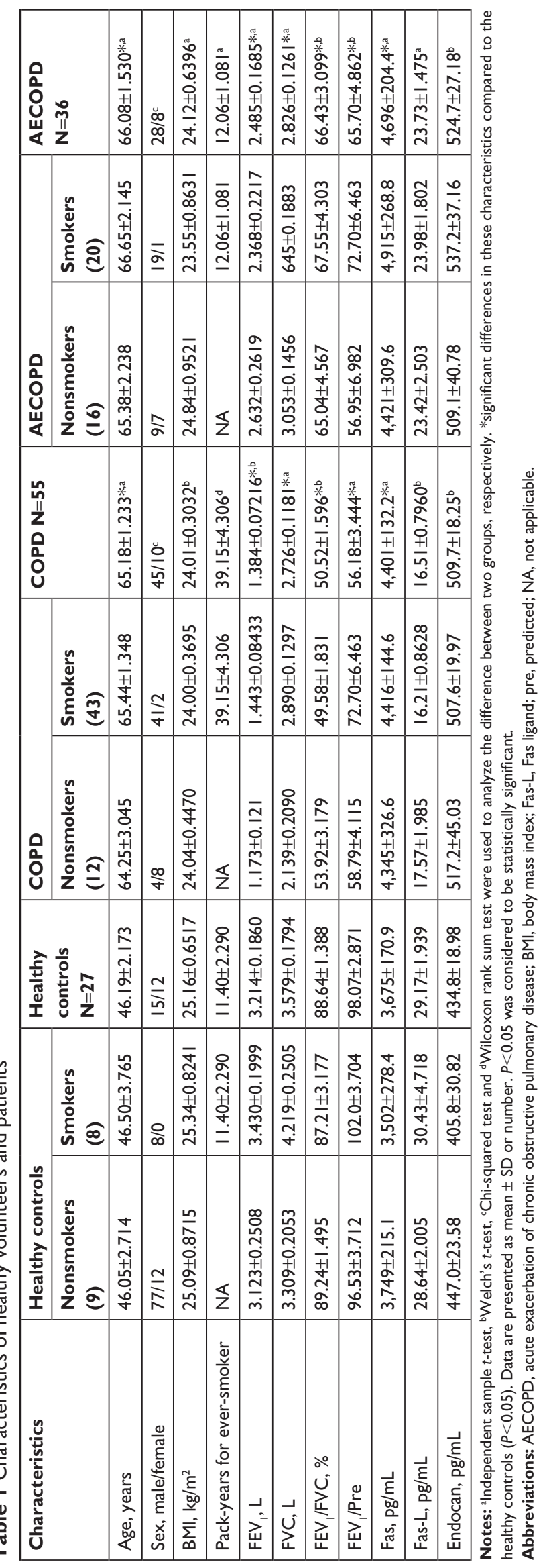



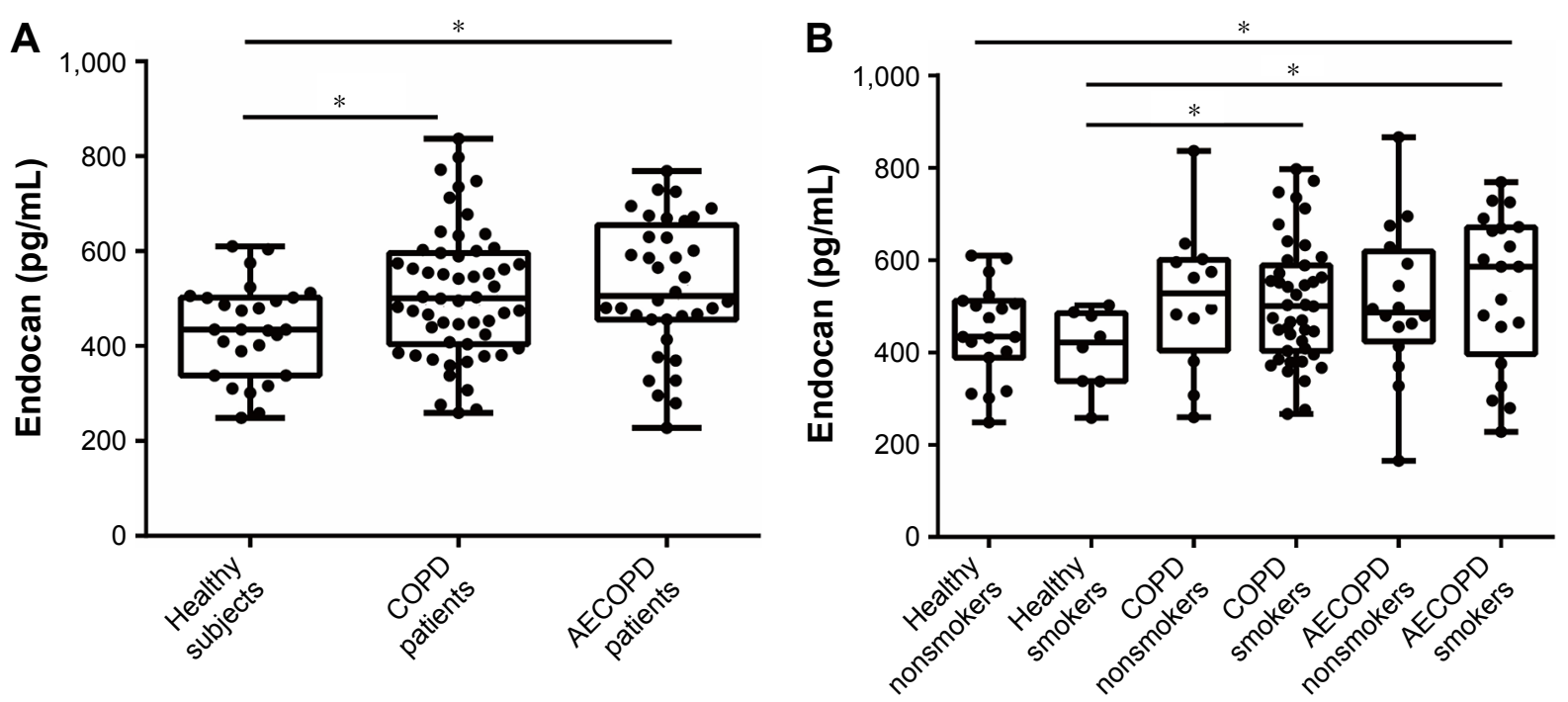

Figure I Plasma endocan levels in healthy, COPD and AECOPD groups.

Notes: (A) Compared with healthy subjects, endocan levels were significantly elevated in COPD and AECOPD patients $(P=0.0124$ and $P=0.0139$, respectively). (B) Endocan levels in COPD and AECOPD smokers were higher than those in healthy smokers $(P=0.0405$ and $P=0.0449$, respectively). AECOPD smokers had a significantly elevated endocan levels when compared with healthy nonsmokers. The difference between healthy smokers and COPD smokers as well as the difference between healthy smokers and AECOPD smokers was analyzed by independent sample $t$-test, and the rest is Welch's $t^{\prime}$ test. $(P=0.049)(* P<0.05)$. Independent sample $t$-test and Welch's $t$ ' test were used. Abbreviation: AECOPD, acute exacerbation of chronic obstructive pulmonary disease.

adapter proteins and forms the death-inducing signaling complex, eventually activating caspase-3, 6, 7 and inducing apoptosis. ${ }^{20,34,35}$ Previous studies have shown that endocan can be involved in cell apoptosis through mitochondrial unfolded protein response and caspase- and $\mathrm{p} 53$-dependent apoptotic pathway. ${ }^{19,20}$ Abnormal structural cell apoptosis in COPD leads to destruction of terminal airways, alveolar walls and pulmonary capillary beds, and is an important mechanism
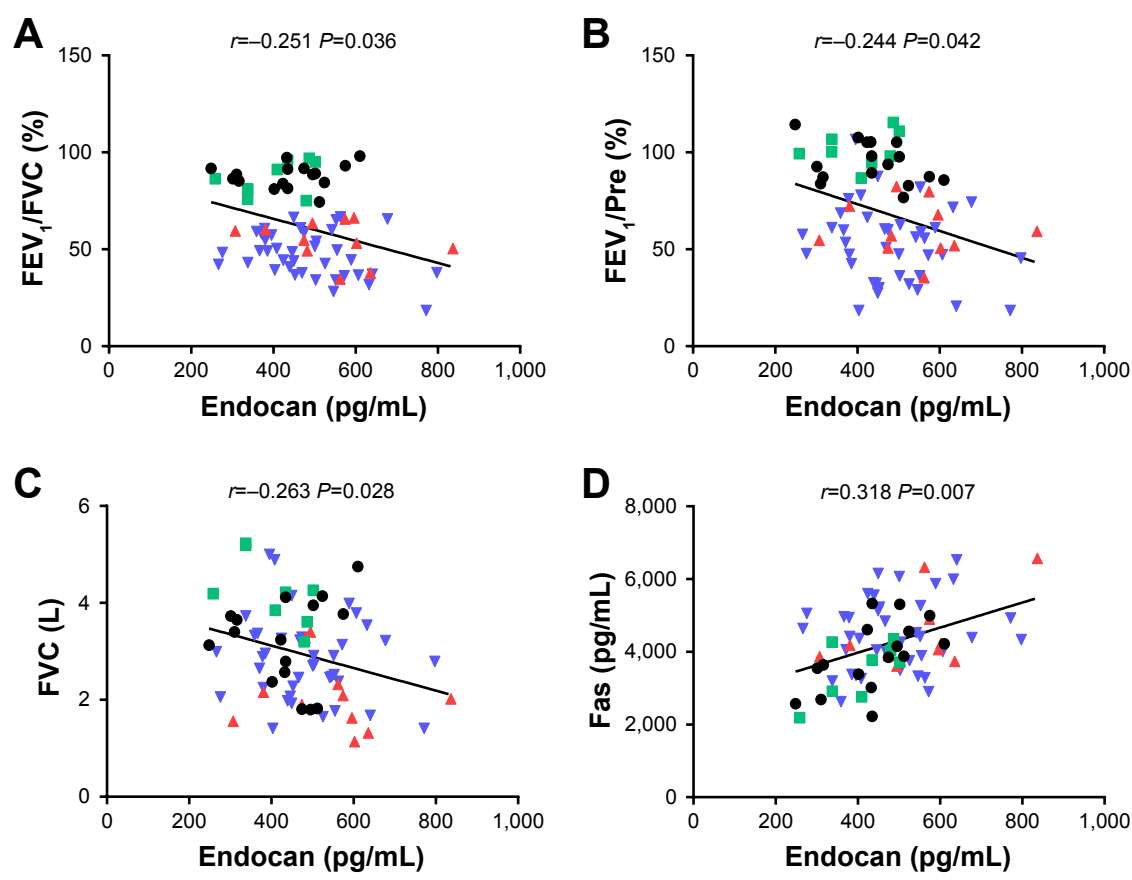

- Healthy nonsmokers $\quad$ Healthy smokers $\triangle$ COPD nonsmokers $\quad$ COPD smokers

Figure 2 Correlations between plasma levels of endocan with FEV /FVC, FEV,/Pre, FVC and Fas in healthy and COPD groups.

Notes: After adjusting for age, gender, BMI and smoking history, endocan was negatively correlated with $F E V_{1} / F V C$ (adjusted $\left.r=-0.25 \mathrm{I}, P=0.036\right)(\mathbf{A})$; FEV,/Pre (adjusted $r=-0.244, P=0.042$ ) (B); FVC (adjusted $r=-0.263, P=0.028)(C)$; and positively correlated with Fas (adjusted $r=0.3 \mid 8, P=0.007)(D)$.

Abbreviations: AECOPD, acute exacerbation of chronic obstructive pulmonary disease; BMI, body mass index; pre, predicted. 

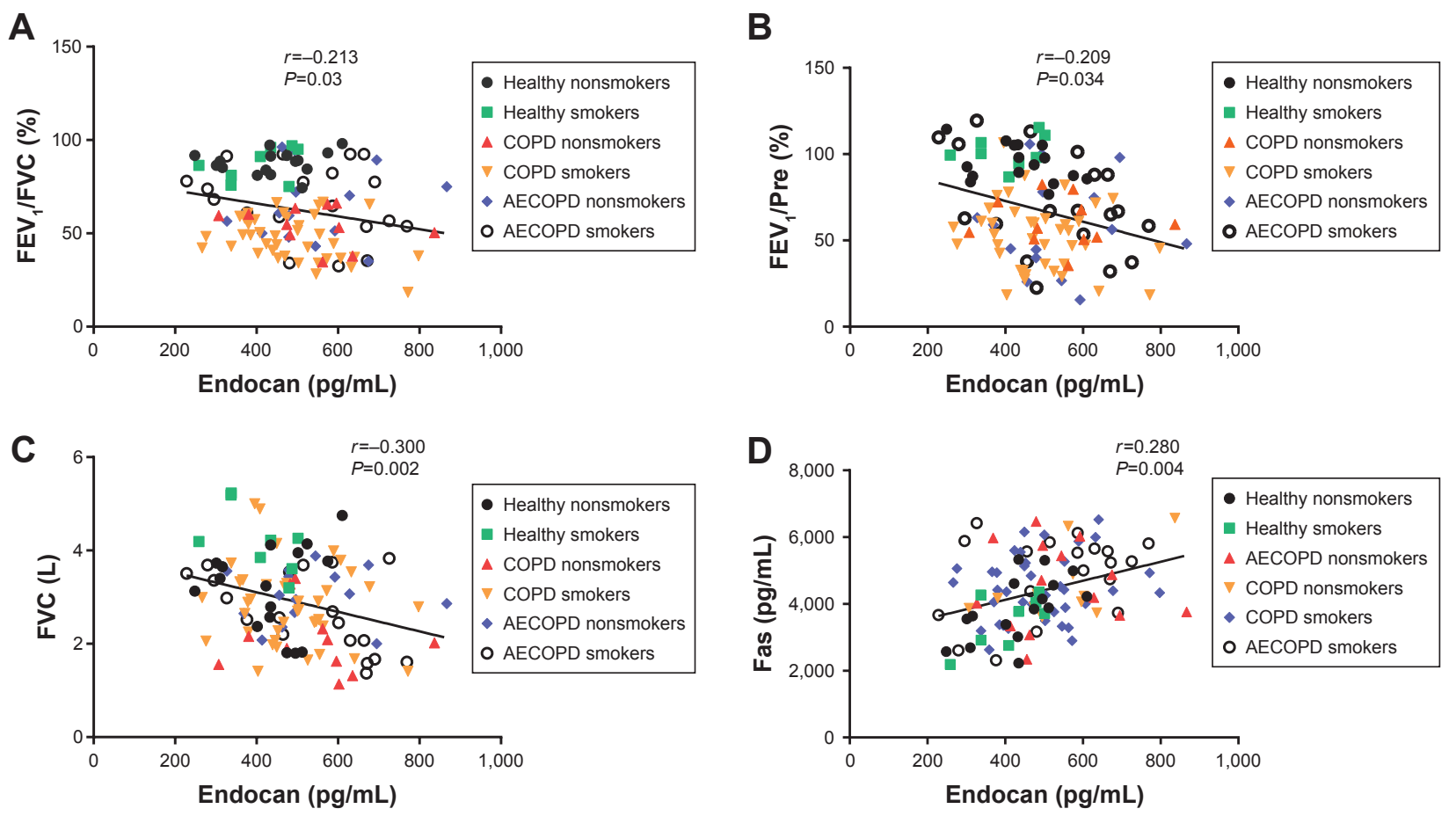

Figure 3 Correlations between plasma levels of endocan with FEV,/FVC, FEV,/Pre, FVC and Fas in healthy, COPD and AECOPD groups.

Notes: After adjusting for age, gender, BMI and smoking history, endocan was negatively correlated with $F E V_{1} / F V C$ (adjusted $\left.r=-0.213, P=0.03\right)(A)$ ) FEV /Pre (adjusted $r=-0.209, P=0.034$ ) (B); FVC (adjusted $r=-0.300, P=0.002)(C)$; and positively correlated with Fas $(r=0.280, P=0.004)(D)$.

Abbreviations: AECOPD, acute exacerbation of chronic obstructive pulmonary disease; BMI, body mass index; pre, predicted.

involved in the development of COPD. ${ }^{36}$ Therefore, we speculated that endocan may be involved in abnormal cell apoptosis in COPD, with abnormal apoptosis leading to damage and remodeling of the lung structure, which in turn affects ventilatory function in COPD.

There are several limitations to this study to be noted. First, because of the strict inclusion and exclusion criteria, we recruited only 118 subjects. The limited number of patients may affect the $P$-value of the correlation analysis and may result in false-negative correlations between endocan and other pulmonary function parameters. Endocan levels in

Table 2 Multiple linear regression analysis for circulating endocan levels

\begin{tabular}{|l|l|l|l|l|}
\hline Parameter & $\boldsymbol{\beta}$ & $\boldsymbol{P}$-value & Tolerance & VIF \\
\hline Age & 0.250 & $0.013^{*}$ & 0.770 & $\mathrm{I} .299$ \\
\hline BMI & 0.086 & $0.34 \mathrm{I}$ & 0.929 & $\mathrm{I} .077$ \\
\hline Sex & -0.065 & 0.543 & 0.648 & $\mathrm{I} .542$ \\
\hline Smoking history & -0.100 & 0.350 & 0.661 & $\mathrm{I} .514$ \\
\hline FEV,/FVC & $-0.27 \mathrm{I}$ & $0.006^{*}$ & 0.787 & $\mathrm{I} .27 \mathrm{I}$ \\
\hline Fas & 0.261 & $0.005^{*}$ & 0.887 & $\mathrm{I} .128$ \\
\hline Fas-L & $0.25 \mathrm{I}$ & 0.013 & 0.763 & $\mathrm{I} .3 \mathrm{II}$ \\
\hline
\end{tabular}

Notes: Age, BMI, gender, smoking history, FEV/FVC, Fas and Fas-L were the independent parameters associated with endocan. The value of $\beta$ represents standard regression coefficient. ${ }^{*} P<0.05$. When the tolerance closes to 0 or $\mathrm{VIF} \geq 10$, serious multicollinearity can be considered.

Abbreviations: BMI, body mass index; Fas-L, Fas ligand; VIF, variance inflation factor.
AECOPD patients were higher than those in COPD patients; however, there was no statistical significance. Perhaps, there will be more discoveries as we expand the sample size. Second, the subjects included in the healthy control group were younger than those in COPD and AECOPD groups. However, we adjusted for age during the analysis and there are no studies showing that age has an important relationship with endocan expression. Ultimately, our study only shows that endocan may be related to COPD, but the specific mechanism remains unknown. Future studies should expand the sample size and focus on the mechanism by which endocan affects COPD.

\section{Conclusion}

Overall, our findings indicate that endocan may be associated with a decrease in ventilatory function in COPD and may be related to apoptosis of structural pulmonary cells, revealing that endocan may play an important role in the pathogenesis of COPD. This work provides new insights into COPD pathogenesis.

\section{Acknowledgments}

This work was supported by grants from the National Key Research and Development Program in China (numbers 2016YFC1303600 and 2016YFC1304500), the National Natural Science Foundation of China (numbers 81470236 
and 81670038), and the Sichuan Science and Technology Support Program (number 2015SZ0151). The funders had no role in study design, data collection and analysis, decision to publish or preparation of the manuscript.

\section{Author contributions}

Luqi Dai and Junyun He designed this research, and all the authors contributed toward subject recruitment, data collection, statistical analysis, and drafting and critically revising the manuscript and all authors gave final approval of the version to be published, and agree to be accountable for all aspects of the work.

\section{Disclosure}

The authors report no conflicts of interest in this work.

\section{References}

1. Barnes PJ. Inflammatory mechanisms in patients with chronic obstructive pulmonary disease. J Allergy Clin Immunol. 2016;138(1): $16-27$.

2. McDonough JE, Yuan R, Suzuki M, et al. Small-airway obstruction and emphysema in chronic obstructive pulmonary disease. $N$ Engl J Med. 2011;365(17):1567-1575.

3. Hillas G, Perlikos F, Tzanakis N. Acute exacerbation of COPD: is it the "stroke of the lungs"? Int J Chron Obstruct Pulmon Dis. 2016;11: 1579-1586.

4. Wedzicha JA, Donaldson GC. Exacerbations of chronic obstructive pulmonary disease. Respir Care. 2003;48(12):1204-1213.

5. Man SF, van Eeden S, Sin DD. Vascular risk in chronic obstructive pulmonary disease: role of inflammation and other mediators. Can J Cardiol. 2012;28(6):653-661.

6. Sampsonas F, Antonacopoulou A, Spathas D, et al. Positive association between two polymorphic sites (+134 insA/delA and G198T) of the endothelin- 1 gene and chronic obstructive pulmonary disease. A case-control study. Respir Med. 2010;104(1):114-120.

7. Polosa R, Malerba M, Cacciola RR, et al. Effect of acute exacerbations on circulating endothelial, clotting and fibrinolytic markers in COPD patients. Intern Emerg Med. 2013;8(7):567-574.

8. Polatli M, Cakir A, Cildag O, Bolaman AZ, Yenisey C, Yenicerioglu Y. Microalbuminuria, von Willebrand factor and fibrinogen levels as markers of the severity in COPD exacerbation. J Thromb Thrombolysis. 2008;26(2):97-102.

9. Roland M, Bhowmik A, Sapsford RJ, et al. Sputum and plasma endothelin-1 levels in exacerbations of chronic obstructive pulmonary disease. Thorax. 2001;56(1):30-35.

10. Delehedde M, Devenyns L, Maurage CA, Vivès RR. Endocan in cancers: a lesson from a circulating dermatan sulfate proteoglycan. Int J Cell Biol. 2013;2013:705027.

11. Kechagia M, Papassotiriou I, Gourgoulianis KI. Endocan and the respiratory system: a review. Int J Chron Obstruct Pulmon Dis. 2016;11: 3179-3187.

12. Pauly D, Hamed S, Behnes M, et al. Endothelial cell-specific molecule-1/ endocan: Diagnostic and prognostic value in patients suffering from severe sepsis and septic shock. J Crit Care. 2016;31(1):68-75.

13. Yilmaz MI, Siriopol D, Saglam M, et al. Plasma endocan levels associate with inflammation, vascular abnormalities, cardiovascular events, and survival in chronic kidney disease. Kidney Int. 2014;86(6): 1213-1220.

14. Cox LA, van Eijk LT, Ramakers BP, et al. Inflammation-induced increases in plasma endocan levels are associated with endothelial dysfunction in humans in vivo. Shock. 2015;43(4):322-326.
15. Lee W, Ku SK, Kim SW, Bae JS. Endocan elicits severe vascular inflammatory responses in vitro and in vivo. J Cell Physiol. 2014; 229(5):620-630.

16. Sarrazin S, Lyon M, Deakin JA, et al. Characterization and binding activity of the chondroitin/dermatan sulfate chain from Endocan, a soluble endothelial proteoglycan. Glycobiology. 2010;20(11): $1380-1388$.

17. Lassalle P, Molet S, Janin A, et al. ESM-1 is a novel human endothelial cell-specific molecule expressed in lung and regulated by cytokines. J Biol Chem. 1996;271(34):20458-20464.

18. Kali A, Shetty KS. Endocan: a novel circulating proteoglycan. Indian J Pharmacol. 2014;46(6):579-583.

19. Zhang X, Zhuang R, Wu H, et al. A novel role of endocan in alleviating LPS-induced acute lung injury. Life Sci. 2018;202:89-97.

20. Sumei Z, Shaolong C, Xiang W, Yinliang Q, Qing Z, Yuan W. Endocan reduces the malign grade of gastric cancer cells by regulating associated protein expression. Tumour Biol. 2016;37(11):14915-14921.

21. Peinado VI, Pizarro S, Barberà JA. Pulmonary vascular involvement in COPD. Chest. 2008;134(4):808-814.

22. Mikkelsen ME, Shah CV, Scherpereel A, et al. Lower serum endocan levels are associated with the development of acute lung injury after major trauma. $J$ Crit Care. 2012;27(5):522.e11-522.e17.

23. Tang L, Zhao Y, Wang D, et al. Endocan levels in peripheral blood predict outcomes of acute respiratory distress syndrome. Mediators Inflamm. 2014;2014:625180-625189.

24. Zhang X, Zhuang R, Wu H, et al. A novel role of endocan in alleviating LPS-induced acute lung injury. Life Sci. 2018;202:89-97.

25. Bokov P, Delclaux C. Interpretation and use of routine pulmonary function tests: Spirometry, static lung volumes, lung diffusion, arterial blood gas, methacholine challenge test and 6-minute walk test. Rev Med Interne. 2016;37(2):100-110.

26. de Torres JP, Casanova C, Montejo de Garcini A, Aguirre-Jaime A, Celli BR. Gender and respiratory factors associated with dyspnea in chronic obstructive pulmonary disease. Respir Res. 2007;8:18.

27. O'Donnell DE, Webb KA. Breathlessness in patients with severe chronic airflow limitation. Physiologic correlations. Chest. 1992;102(3): $824-831$.

28. Mahut B, Chevalier-Bidaud B, Plantier L, et al. Diffusing capacity for carbon monoxide is linked to ventilatory demand in patients with chronic obstructive pulmonary disease. COPD. 2012;9(1):16-21.

29. Zavorsky GS, van der Lee I. Can the measurement of pulmonary diffusing capacity for nitric oxide replace the measurement of pulmonary diffusing capacity for carbon monoxide? Respir Physiol Neurobiol. 2017;241:9-16.

30. Balta S, Mikhailidis DP, Demirkol S, Ozturk C, Celik T, Iyisov A. Endocan: A novel inflammatory indicator in cardiovascular disease? Atherosclerosis. 2015;243(1):339-343.

31. Sarrazin S, Adam E, Lyon M, et al. Endocan or endothelial cell specific molecule-1 (ESM-1): a potential novel endothelial cell marker and a new target for cancer therapy. Biochim Biophys Acta. 2006;1765(1): 25-37.

32. Moro L, Pedone C, Scarlata S, Malafarina V, Fimognari F, AntonelliIncalzi R. Endothelial dysfunction in chronic obstructive pulmonary disease. Angiology. 2008;59(3):357-364.

33. Hashimoto M, Tanaka H, Abe S. Quantitative analysis of bronchial wall vascularity in the medium and small airways of patients with asthma and COPD. Chest. 2005;127(3):965-972.

34. Stoneman VE, Bennett MR. Role of Fas/Fas-L in vascular cell apoptosis. J Cardiovasc Pharmacol. 2009;53(2):100-108.

35. Yonehara S, Ishii A, Yonehara M. A cell-killing monoclonal antibody (anti-Fas) to a cell surface antigen co-downregulated with the receptor of tumor necrosis factor. $J$ Exp Med. 1989;169(5):1747-1756.

36. Houghton AM. Mechanistic links between COPD and lung cancer. Nat Rev Cancer. 2013;13(4):233-245. 


\section{Publish your work in this journal}

The International Journal of COPD is an international, peer-reviewed journal of therapeutics and pharmacology focusing on concise rapid reporting of clinical studies and reviews in COPD. Special focus is given to the pathophysiological processes underlying the disease, intervention programs, patient focused education, and self management protocols.

This journal is indexed on PubMed Central, MedLine and CAS. The manuscript management system is completely online and includes a very quick and fair peer-review system, which is all easy to use. Visit http://www.dovepress.com/testimonials.php to read real quotes from published authors.

Submit your manuscript here: http://www.dovepress.com/international-journal-of-chronic-obstructive-pulmonary-disease-journal 